\title{
Energieverbrauchsmessung haustechnischer Komponenten mittels Feldbussen der Gebäudeautomatisierung
}

Schwerpunkt: B9, B3

Dipl.-Ing. Patrick Diekhake,

Dipl.-Ing. Tamás Kurczveil,

M.Sc. Jieyu Liu,

Prof. Dr.-Ing. Dr. h.c. mult. Eckehard Schnieder,

Institut für Verkehrssicherheit und Automatisierungstechnik, Technische Universität Braunschweig

Tel.: +49-531-391-3317

\{diekhake, kurczveil, liu, schnieder\}@iva.ing.tu-bs.de

\section{Einführung}

Energie ist eine der wichtigsten Grundlagen moderner Gesellschaften, deren verantwortliche Nutzung aufgrund begrenzter Ressourcen immer dringlicher wird. Insbesondere in Gebäuden lässt sich der Energiebedarf durch intelligente Lösungen deutlich verringern. Grundlage dieser Lösungen wie beispielsweise ein Lastmanagement zur optimierten Energieverteilung ist die Energiemessung jeder einzelnen Verbrauchs-Komponente in dem System. In diesem Beitrag soll ein Feldbussystem vorgestellt werden, welches sich aufgrund seiner örtlichen Verteilung für die Energieverbrauchsmessung einzelner Komponenten eignet. Das Konzept wird dargestellt, mathematisch beschrieben und ein Beispiel aufgezeigt.

\section{Feldbussystem SmallCAN}

SmallCAN ist ein optimiertes Feldbussystem, welches am Institut für Verkehrssicherheit und Automatisierungstechnik entwickelt wurde und sich u.a. für die Gebäudeautomatisierung (GA) eignet. Die Optimierung beinhaltet unter anderem die Verwaltung hoher Teilnehmerzahlen von bis zu 1000 Buskopplern sowie eine räumliche Ausdehnung der Bus-Leitung auf eine Länge von bis zu 1000m.

Ein wesentliches Ziel von SmallCAN ist dessen vollständige Dezentralisierung um erhöhten Kabelaufwand und hohe Kosten für die Auslegung einer Zentraleinheit zu vermeiden. Die konsequente Verfolgung dieses Ziels erfordert die Ausstattung eines jeden Aktors und Sensors mit einem eigenen (integrierten) Buskoppler. Mit diesem Ansatz können Insellösungen vermieden und die einzelnen Geräte an die jeweilige Situation angepasst werden. Neben einer universellen Erweiterbarkeit und Übersichtlichkeit bietet die dezentrale Struktur ebenfalls den Vorteil der Zustandserfassung einzelner Geräte (z.B. Verbraucher). Somit stellt das SmallCAN-Feldbussystem ein umfangreiches System aus intelligenten Sensoren dar, welches die Energieverbrauchsmessung einzelner, an das System gekoppelten Komponenten erlaubt.

Die dezentrale Verteilung des Feldbussystems stellt jedoch einige Anforderungen an den Buskoppler. Die erhöhte Busteilnehmerzahl setzt den Einsatz eines preisgünstigen, energiesparenden und universell einsetzbaren Buskopplers voraus: Die Busankopplung bei SmallCAN basiert auf dem sparsamen robusten Mikrocontroller PIC16F88. Der Bustransceiver besteht aus einem MOSFET des Typs BS170. Der Receiver besteht lediglich aus einem Spannungsteiler. Der über das Buskabel versorgte LDOSpannungsregler beliefert den PIC16F88 mit einer konstanten Spannung und vervollständigt den gering bestückten und preiswerten Buskoppler. Für die hardwaretechnische Anpassung an die anzuschließende Gerätekomponente wird ein Anwendungsadapter verwendet. In Kombination mit dem einheitlichen integrierbaren Buskoppler kann die Gerätekomponente so zur Bustauglichkeit überführt werden. Der Eigenverbrauch eines Anwendungsmoduls inklusiv aufgesteckten Buskoppler beträgt gerade $80 \mathrm{~mW}$ sodass eine Energiespeisung in das gesamte System auch bei einer hohen Teilnehmeranzahl über ein zentrales Netzteil möglich und der Betrieb des GA-Systems wirtschaftlich ist. 
Auch die Investitionskosten für ein Anwendungsmodul sind im Systementwurf niedrig gehalten. Die SmallCAN-Komponenten bestehen aus kostengünstigen und bewährten Standardbauteilen und handelsüblichen Gehäusen, die keine zusätzlichen Spezialanfertigungen voraussetzen. Die kostengünstig bereitgestellten Busteilnehmer können in einfacher Bus-Topologie flexibel verbunden werden. Als Busmedium wird herkömmliches geschirmtes Telefonkabel (Stern-Vierer) verwendet.

Die Software des Buskopplers besteht aus folgenden wesentlichen modularen Komponenten:

- Betriebssystem

- Sonderfunktion

- Freie Sonderfunktionen

Das Betriebssystem (BS) des Buskopplers enthält alle zur Buskommunikation notwendigen Routinen. Sonderfunktionen (SF) steuern und kontrollieren die auf dem Applikationsadapter befindliche Hardware. An dieser Stelle kann die eigentliche Funktion des Busteilnehmers beziehungsweise des Applikationsadapters programmiert werden. Freie Sonderfunktionen (FSF) sind unabhängig von der Hardware und führen bei Bedarf eine weitergehende und übergreifende Verarbeitung der per Bus bereitgestellten Daten in allgemeiner Form durch. Dazu gehört u. a. das Vergleichen, Skalieren und Zusammenführen von Daten sowie Regelalgorithmen, wie beispielsweise eine Klimasteuerung. Da die FSF hardwareunabhängig sind, können sie dezentral in einen beliebigen Buskoppler mit ausreichend vorhandenen Speicherressourcen ausgelagert werden.

Ist das SCANSystem an einen PC-Server angeschlossen, lässt es sich via LAN-Netzwerkverbindung komfortabel an ein Software-Tool anbinden. Wesentliche Aufgaben wie Konfiguration, Parametrierung, Visualisierung, Programmierung, Netzwerkmanagement und Applikationsverwaltung erfolgen über dieses Tool. Die Software besteht aus dem SmallCANserver, der die Anbindung an das SCAN-System über eine serielle RS232 Schnittstelle bereitstellt, und dem eigentlichen SmallCANtool. Zur Konfiguration des Systems unterstützen Konfigurationsdialoge des SmallCANtools mithilfe derer die Sende- und Empfangsadressen der Busnachrichten in den EEPROM Speicher des jeweiligen Buskopplers geschrieben werden. So sind Funktionsänderungen, Erweiterungen und Anpassungen des Systems einfach zu realisieren. Zur Visualisierung der Daten können in einer GUI-Umgebung Visualisierungselemente geladen werden, wobei eine Anordnung der Elemente entsprechend der realen räumlichen Verhältnisse über eine beliebige Bilddatei möglich ist.

\section{Konzept zur Energieverbrauchsmessung}

Das Konzept zur Energieverbrauchsmessung einzelner Komponenten ist in Abbildung 1 dargestellt. Zunächst wird die aktuelle Gesamtleistung aller Komponenten über einen Energiezähler ermittelt. Die aktuelle Leistungsaufnahme aller Geräte sowie der Gesamtenergieverbrauch werden von dort als Nachrichtentelegramme auf dem Bus abgelegt. 


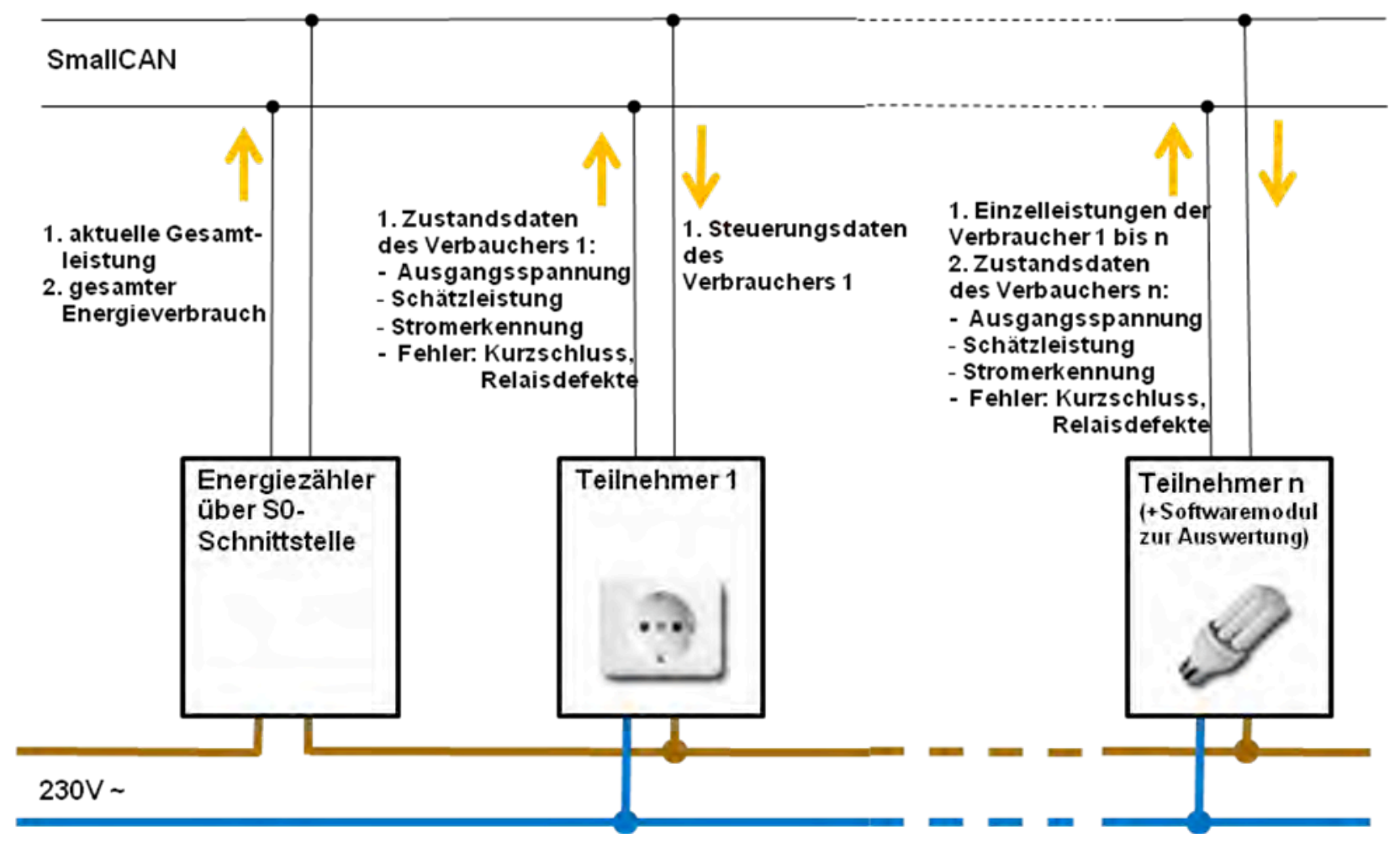

Abbildung 1: Konzept zur Energieverbrauchsmessung einzelner Komponenten

Über das örtlich verteilte Feldbussystem SmallCAN können die Gerätekomponenten (Verbraucher) angesteuert werden. Weiter werden deren Zustände erfasst, zum Beispiel ob das Gerät ein oder ausgeschaltet ist oder Hardwaredefekte vorliegen. Die Funktionen zur Ansteuerung der Gerätekomponenten und dessen Zustandserfassung werden von den hardwareabhängigen Sonderfunktionen ausgeführt, die die Datentelegramme zur Ansteuerung der Gerätekomponente vom Bus empfangen und die Zustandsinformationen als Datentelegramme auf dem Bus zur Verfügung stellen.

Zur Auswertung wird eine Freie Sonderfunktion verwendet, die alle zur Verarbeitung benötigten Daten vom Bussystem einliest. Dieses Softwaremodul kann dezentral in einen beliebigen Buskoppler geladen werden (hier Buskoppler von Teilnehmer $n$ ). Die Freie Sonderfunktion ermittelt die Einzelleistungen der jeweiligen Verbraucher und stellt diese Information wiederum auf dem Bussystem zur Verfügung. Über eine PC-Anbindung des SmallCAN Systems lassen sich die ermittelten Einzelleistungen visuell darstellen.

Alle zur Auswertung relevanten Nachrichtenadressen werden in die Empfangsliste des Buskopplers, der die Freie Sonderfunktion beinhaltet, eingetragen. Die Adressen der von der Freien Sonderfunktion zu versendenen Telegramme stehen in einer entsprechenden Sendeliste.

Bei einer erhöhten Anzahl von systemgebundenen Verbrauchern (bis zu 1000 Teilnehmer) kann aufgrund der notwendigen rechen- und speicheraufwendigen Prozesse die Freie Sonderfunktion als Prozesssonderfunktion auf den Server-PC ausgelagert werden.

\section{SmallCAN Anwendungsadapter}

Zur Realisierung des Konzeptes bedarf es neben einem Anwendungsadapter zur Auswertung eines Energiezählers auch die Erweiterungen bereits vorhandener Anwendungsadapter mit bestimmter Hardware.

Zur Auswertung des Wechselstrom-Energiezählers ist ein Anwendungsadapter entwickelt worden, der die von der S0 Schnittstelle gelieferten Stromimpulse einliest. Diese werden von der auf dem Buskoppler installierten Sonderfunktion innerhalb einer festen Zeitdauer gezählt. Mit der Rückrechnung der Zählerkonstante (beispielsweise 2000 Impulse pro kWh) wird die aktuelle Leistungsaufnahme der an das SmallCAN System angeschlossenen Verbraucher ermittelt. 
Zur Ansteuerung der einzelnen Verbraucher und deren Zustandserfassung sind schaltungstechnische Lösungen einzubinden die am Ort der Gerätekomponente auf dem Anwendungsadapter realisiert werden. Folgende Funktionen sind somit an jedem Verbraucher durch die Sonderfunktion ausführbar:

- Der Verbraucher wird über ein optoelektrische Relais geschaltet.

- Die Überwachung der 230V Versorgungsspannung erfolgt über Wechselspannungs-Optokoppler. Weiter wird die Ausgangsspannung des optoelektrischen Relais geprüft. Dadurch können Relaisdefekte detektiert werden.

- Es muss weiter abgesichert sein, das kein Verbraucherdefekt vorliegt. Dazu wird der Ausgang des Schaltrelais auf Kurzschluss und Stromfluss überwacht. Die Detektion eines Kurzschlusses erfolgt anhand der Spannung über einem Vorwiderstand. Bei Überschreitung eines maximalen Spannungswertes wird eine Fehlermeldung auf dem Bus generiert. Die Stromerkennung erfolgt über einem Messwiderstand der bei Stromfluss einen Spannungsabfall erzeugt. Dieser wird zur Vermeidung erhöhter Wärmeentwicklung durch Schottky-Leistungsdioden begrenzt. Der geringe Spannungsabfall steuert einen „vorgespannten“ Transisor, der wiederum einen Optokoppler schaltet. Das Ausgangssignal des Optokopplers wird von einem Eingangs-Pin des Busskopplers eingelesen und über die Sonderfunktion ausgewertet. Somit ist die Funktionskontrolle der geschalteten Geräte ist durch die Erkennung des Stromflusses möglich. Wird bei eingeschalteten Relais kein Stromfluss im Verbraucher erkannt, wird ein No-Current Fehler generiert.

- Über die Sonderfunktion kann weiter innerhalb einer Halbwelle die Zeit gemessen werden, in der die Stromerkennung aktiv ist. Damit sind ein Rückschluss auf die elektrische Leistung (ohmsche Last) und ein Vergleich zum geeichten Energiezähler möglich, wobei der über die Stromflusserkennung ermittelte Leistungswert aufgrund von Nichtlinearitäten nur als grober Schätzwert zu berücksichtigen ist.

\section{Mathematische Berechnung der Leistungsaufnahme einzelner Verbraucher}

Ist ein SmallCAN-GA-System mit den notwendigen Komponenten zur Energiemessung ausgestattet, wird die Berechnung der Leistungsaufnahme einzelner Komponenten durch einen Zustandswechsel initiiert (Ab- bzw. Zuschalten eines Verbrauchers) und in zwei Schritten ausgeführt.

Der Zustandsvektor $\underline{Z}[k]$ beschreibt den vorherrschenden Systemzustand zum diskreten Zeitpunkt $k$, dessen Elemente $Z_{i=1 \mathrm{~K}}[k]$ nur binäre Werte annehmen können, um die Zustände aller Verbraucher (an bzw. aus) zum Zeitpunkt $k$ zu beschreiben. Die Anzahl der Elemente $n$ ist somit gleich mit der Anzahl angeschlossener elektrischer Verbraucher.

Für $n$ Verbraucher ergeben sich $2^{n}$ mögliche Kombinationen für den Zustandsvektor ${ }^{j=1 K} 2^{n} \underline{Z}$. Mit dem Ziel, die mittlere Leistungsaufnahme einzelner Verbraucher zu ermitteln, müssen die Zustandsvektoren zusätzlich mit den notwendigen Daten zur Energieaufnahme pro Zeiteinheit versehen werden. Um eine vollständige Erfassung zu ermöglichen wird in einem ersten Schritt die Systemmatrix $\underline{S}$ definiert, die den benötigten Speicherplatz auf dem verwendeten Mikrokontroller widerspiegelt und jedem möglichen Systemzustand eine summierte Verweildauer ${ }^{j} T$ und die Summe der aufgenommenen Energie ${ }^{j} E$ zuweist. Die Systemmatrix $\underline{S}$, mit der diese Informationen für alle Systemzustandsmöglichkeiten erfasst werden können, ist in Gleichung (1) dargestellt. Die agglomerierte Zustandsmatrix $\underline{Z}_{A}$ stellt hierbei alle möglichen Zustandskombinationen dar, die Verweildauer in den zugehörigen Zuständen ${ }^{j} \underline{Z}$ wird im Vektor $\underline{T}$ und die aufgenommene Energie in $\underline{E}$ erfasst. 


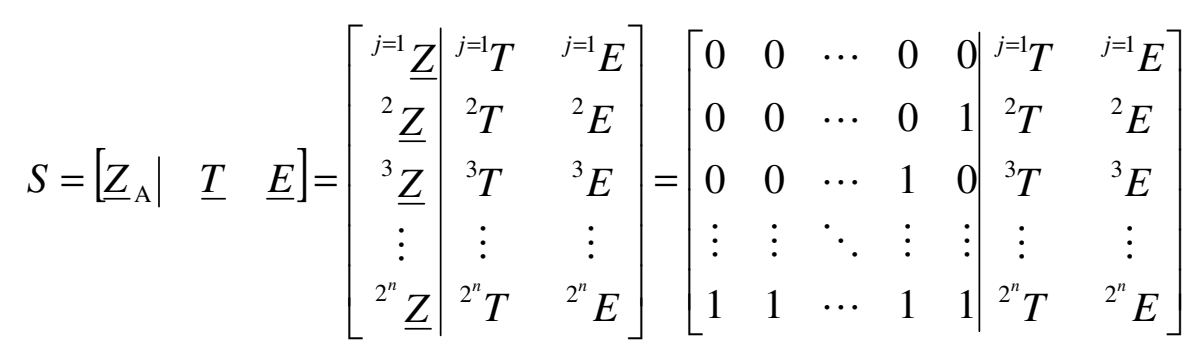

Es kann somit Gleichungssystem (2) aufgestellt werden, um mit dessen Lösung die Leistungsaufnahme aller Verbraucher $\underline{P}$ zu berechnen.

$$
\underline{Z}_{\mathrm{A}} \cdot \underline{P}=\left(\begin{array}{c}
{ }^{j=1} \underline{\underline{Z}} \\
{ }^{2} \underline{\underline{Z}} \\
\underline{\underline{Z}} \\
\underline{\underline{Z}} \\
\vdots \\
{ }^{2^{n}} \underline{\underline{Z}}
\end{array}\right) \cdot \underline{P}=\left(\begin{array}{ccccc}
0 & 0 & \cdots & 0 & 0 \\
0 & 0 & \cdots & 0 & 1 \\
0 & 0 & \cdots & 1 & 0 \\
\vdots & \vdots & \ddots & \vdots & \vdots \\
1 & 1 & \cdots & 1 & 1
\end{array}\right) \cdot\left(\begin{array}{c}
P_{i=1} \\
\vdots \\
P_{n}
\end{array}\right)=\left(\begin{array}{c}
{ }^{j=1} E /{ }^{j=1} T \\
{ }^{2} E /{ }^{2} T \\
{ }^{3} E /{ }^{3} T \\
\vdots \\
{ }^{2^{n}} E /{ }^{2^{n}} T
\end{array}\right)
$$

Nimmt man an, dass alle Verbraucher eine Leistungsaufnahme $P_{i}>0 \mathrm{~W}$ besitzen, muss aufgrund der nichtnegativen Koeffizientenmatrix, die durch die agglomerierte Zustandsmatrix $\underline{Z}_{A}$ beschrieben wird, für die nichttriviale Lösung $\underline{x} \neq \underline{0}$ ein inhomogenes Gleichungssystem $\underline{A} \underline{x}=\underline{b}$ vorliegen. Dieses kann aus der Systemmatrix extrahiert werden, indem alle Zeilen der Systemmatrix $\underline{S}$ berücksichtigt werden, für deren Einträge ${ }^{j} T \neq 0$ gilt. Dies resultiert in der reduzierten Systemmatrix $\underline{S}_{\text {red }}$ aus welcher die Daten zur Leistungsaufnahme aller Verbraucher über den Gauß-Jordan-Algorithmus berechnet werden können, wenn gilt

$$
\operatorname{Rang}\left(\begin{array}{c}
\vdots \\
{ }^{j} \underline{Z}\left({ }^{j} T \neq 0\right) \\
\vdots
\end{array}\right)=\operatorname{Rang}\left(\underline{Z}_{\mathrm{A}, \text { red }}\right)=n .
$$

Diese Art der Datenerfassung ermöglicht zwar die Ermittlung der Leistungsaufnahme einzelner Komponenten, stellt die Informationen jedoch in Anbetracht des überbestimmten Gleichungssystems (2) (und somit des exponentiellen Zusammenhangs) zu Kosten des Speicheraufwands redundant zur Verfügung. Mit der Annahme, dass 4 Bytes je Variable ${ }^{j} T$ und ${ }^{j} E$ in Anspruch genommen werden, und unter Vernachlässigung der reduzierten Zustandsmatrix $\underline{Z}_{\mathrm{A} \text {,red }}$, berechnet sich der benötigte Speicher zu

$$
M(n)=2^{n} \cdot 2 \cdot 4 \text { Byte. }
$$

Da viele GA-Systeme sich jedoch durch eine hohe Anzahl an Verbrauchern auszeichnen, soll in diesem Abschnitt eine Methode vorgestellt werden, die zur Berechnung der Leistungsaufnahme aller individuellen Verbraucher einen linear von der Verbraucherzahl abhängigen Speicher benötigt. Der Ansatz besteht darin, Linearkombinationen in der reduzierten Systemmatrix $\underline{S}_{\text {red }}$ bzw. reduzierten Zustandsmatrix $\underline{Z}_{\text {A,red }}$ zu vermeiden. Der Rang der reduzierten Zustandsmatrix bleibt somit unverändert $\underline{Z}_{\mathrm{A}, \text { red }} \leq n$, die Anzahl der Zeilen der Systemmatrix $\underline{S}_{\text {red }}$ wird jedoch auf maximal $n$ reduziert.

Die anfängliche Speicherzuweisung erfolgt in Abhängigkeit des Systemabbildes, und reserviert Speicher für eine reduzierten Systemmatrix $\underline{S}_{\text {red }}$, die die gleiche Anzahl von Zeilen $n$ besitzt, wie Verbraucher im System vorhanden sind. Diese wird während der Ausführung fortlaufend mit Daten gefüllt, aus denen sich 
die Leistungsaufnahme berechnen lässt. Die Vektoren $\underline{T}$ und $\underline{E}$ werden Nullvektoren und die reduzierte Zustandsmatrix somit dem Rang Null initiiert.

\section{Schritt 1:}

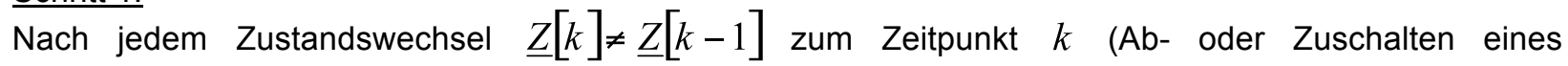
Verbrauchers) erfolgt eine Inkrementierung (beginnend mit dem Wert 0 ) der Hilfsvariablen zur aufgenommenen Energie ${ }^{\text {temp }} \underline{E}$ und Verweildauer ${ }^{\text {temp }} \underline{T}$. Der binäre Hilfsvektor ${ }^{\text {temp }} \underline{Z}=\underline{Z}[k]$ dient zur Kennzeichnung des Zustandes zum aktuellen Zeitpunkt $k$. Nach einem erneuten Zustandswechsel werden diese Variablen nur dann an die reduzierte Systemmatrix $\underline{S}_{\text {red }}$ angehängt, falls sich dadurch eine Rangsteigerung erreichen lässt.

$$
\operatorname{Rang}\left[\underline{Z}_{\text {A,red }}{ }^{\text {temp }} \underline{Z}\right]>\operatorname{Rang}\left(\underline{Z}_{\text {A,red }}\right) \rightarrow \underline{S}_{\text {red,neu }}=\left[\begin{array}{lll} 
& \underline{S}_{\text {red }} \\
\hline{ }^{\text {temp }} \underline{Z} & { }^{\text {temp }} T & { }^{\text {temp }} E
\end{array}\right]
$$

Somit wird sichergestellt, dass nur neue Informationen zur Leistungsaufnahme von Verbrauchern in der reduzierten Systemmatrix $\underline{S}_{\text {red }}$ akkumuliert werden. Diese Anreicherung erfolgt solange, bis der höchstmögliche Rang $n$ erreicht ist. Kann durch die angehängten Hilfsgrößen keine Rangsteigerung erzielt werden, können die somit redundant verfügbaren Verbrauchsdaten mit einer entsprechenden Filterung zur Validierung bisher ermittelter Werte genutzt werden, deren Betrachtung eine Grundlage für zukünftige Arbeiten darstellt.

Schritt 2:

In einem zweiten Schritt werden über den Gauß-Jordan-Algorithmus die Daten zur Leistungsaufnahme berechnet. Für den Fall, dass die reduzierte Systemmatrix $\underline{S}_{\text {red }}$ bereits vollständig gefüllt ist, und somit den Rang $n$ besitzt, können zu jedem der insgesamt $n$ Verbraucher Daten zur Leistungsaufnahme ermittelt werden. Falls der vollständige Rang $n$ nicht erreicht ist, kann dies zwei Gründe haben:

1. mindestens ein Verbraucher wurde seit Betriebsbeginn noch nicht ab- bzw. zugeschaltet oder

2. mindestens zwei Verbraucher wurden seit Betriebsbeginn nur zeitgleich ab- bzw. zugeschaltet.

Beide Fälle zeichnen sich dadurch aus, dass das resultierende Gleichungssystem unterbestimmt ist und

$$
\operatorname{Rang}\left(\underline{Z}_{\mathrm{A}, \mathrm{red}}\right)=m<n
$$

gilt. Somit können nur Daten zu $m$ Verbraucher(-kombinationen) ermittelt werden. Im ersten Fall besitzen in der reduzierten Zustandsmatrix alle Zustandsvektoren ${ }^{j=\mathrm{KK} m} \underline{Z}_{\mathrm{GJ}}$ nach dem Gauß-JordanAlgorithmus jeweils nur ein Element mit dem Wert 1, es treten jedoch auch Nullspalten auf und das Gleichungssystem ist unterbestimmt. Im zweiten Fall besitzt mindestens ein Zustandsvektor ${ }^{j} \underline{Z}_{\mathrm{GJ}}$ nach dem Gauß-Jordan-Algorithmus mehr als ein Element mit dem Wert 1, wodurch das Gleichungssystem ebenfalls unterbestimmt ist. Die zugehörigen Verbraucher müssen dann zu einem Ersatz-Verbraucher reduziert werden, bis zum Zeitpunkt an dem einer der Verbraucher zeitlich getrennt vom anderen geschaltet wird. Gleichungen (7) und (8) verdeutlichen beispielhaft den Aufbau resultierender Gleichungssysteme. 
Fall 1:

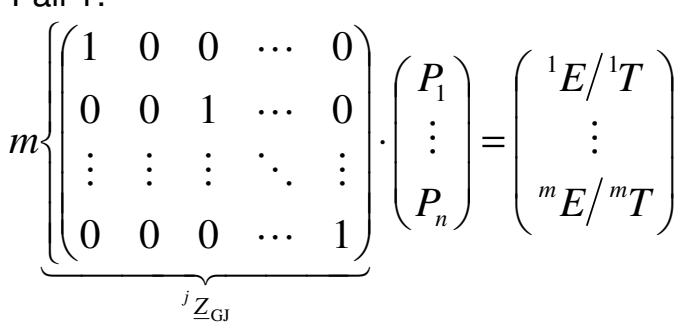

$\rightarrow{ }^{2} P$ unbekannt, weil noch nicht geschaltet

Fall 2:

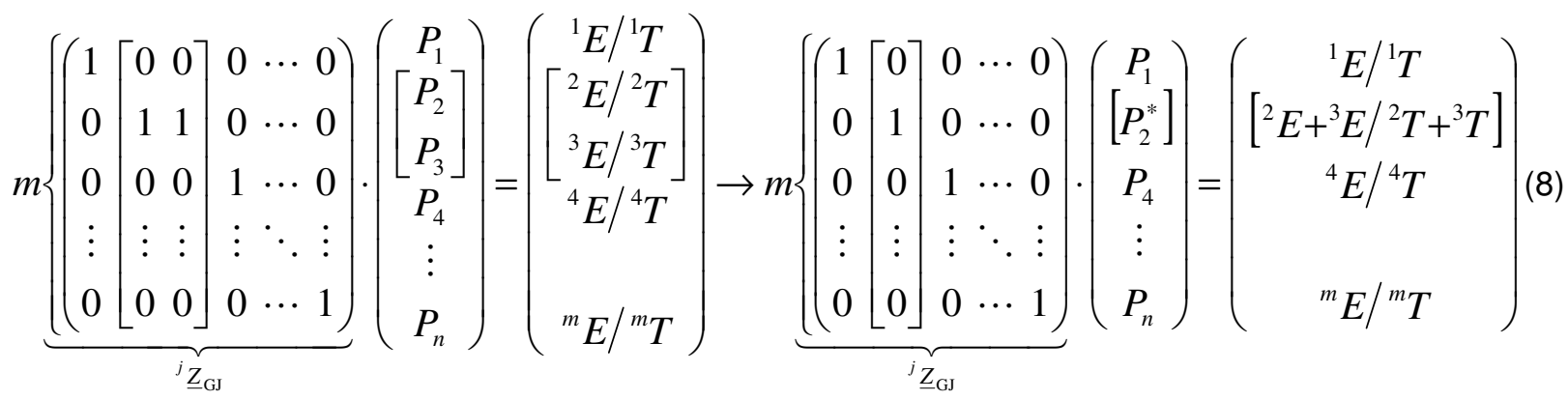

$$
\begin{aligned}
& \rightarrow P_{2}+P_{3}=P_{2}^{*} \text {, da bisher nur zeitgleich geschaltet }
\end{aligned}
$$

Mit laufender Betriebsdauer wird somit die initialisierte Systemmatrix $\underline{S}_{\text {red }}$ mit Informationen angereichert, aus welcher Daten zur Leistungsaufnahme einzelner Verbraucher im beschriebenen zweistufigen Verfahren berechnet werden können.

\section{Beispielhafte Praxisanwendung}

Im Folgenden soll ein Anwendungsbeispiel für ein minimales SmallCAN Feldbussystem zur Ansteuerung, Überwachung und Leistungsmessung von acht Verbrauchern vorgestellt werden. Der Testaufbau besteht aus folgenden Einheiten:

- eine Anwendungsadapter zur Anbindung eines Server-PCs und zur zentralen Energieeinspeisung mit angeschlossenem Netzteil

- ein Server-PC mit der Bediensoftware SmallCAN-Tool

- ein Anwendungsmodul mit Buskoppler zur Ansteuerung der acht Gühlampen

- eine 230V Energieversorgung für die Gühlampen

- ein Anwendungsmodul mit Buskoppler zur Auswertung des Energiezählers, der den Gesamtenergieverbrauch der Gühlampen registriert.

Über die serielle RS232-Schnittstelle der zentralen Energieeinspeisung wird ein PC an das SmallCAN System angebunden.

Abbildung 2 zeigt einen Ausschnitt der Visualisierung des Testaufbaus über das SmallCAN-Tool. Über die dargestellten Schalterelemente können die einzelnen Verbraucher ein- bzw. ausgeschaltet werden. Sämtliche Messdaten wie die Gesamtleistungsaufnahme und die von der Freien Sonderfunktion ermittelten Einzelleistungen werden über Elemente zur Anzeige von Messwerten dargestellt. 

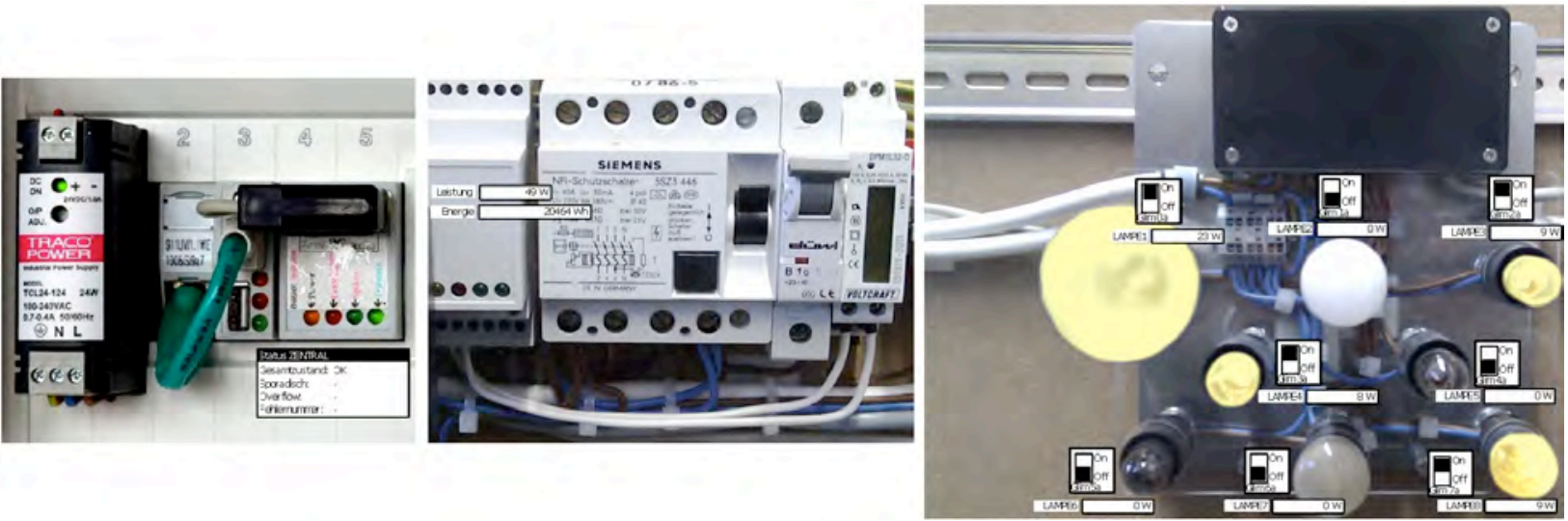

Abbildung 2: Visualisierung der SmallCAN Schnittstelle (li.), des intelligenten Energiezählers (mi.) und der für den Versuchsaufbau relevanten Verbraucher (re.) im SmallCAN-Tool

In Abbildung 2 (re.) werden beispielhaft die berechneten Leistungsaufnahmen der Verbraucher LAMPE1 $\left(P_{\text {LAMPE }}=23 \mathrm{~W}\right)$ LAMPE3 $\left(P_{\text {LAMPE3 }}=9 \mathrm{~W}\right)$ LAMPE4 $\left(P_{\text {LAMPE4 }}=8 \mathrm{~W}\right)$ und LAMPE8 $\left(P_{\text {LAMPES }}=9 \mathrm{~W}\right)$ dargestellt, die sich für den beschriebenen Versuchsaufbau ergeben haben. Ermittelt wurden die Daten über bekannte Systemzustandswechsel und der Kenntnis zum Gesamtverbrauch des Systems, der beispielhaft in Abbildung 2 (mi.) zu sehen ist $\left(P_{g e s}=49 \mathrm{~W}\right)$. Für die übrigen Verbraucher stehen zu diesem Zeitpunkt noch keine Verbrauchsdaten zur Verfügung, da sie bisher weder an- noch ausgeschalten wurden. Abbildung 3 verdeutlicht einen möglichen weiteren Ablauf, in dem die Leistungsaufnahme zweier Verbraucher über deren Zustandsänderungen $\left({ }^{j} Z \rightarrow{ }^{j+1} Z \rightarrow{ }^{j+2} Z\right)$ mit den vorgestellten Algorithmen berechnet werden kann.

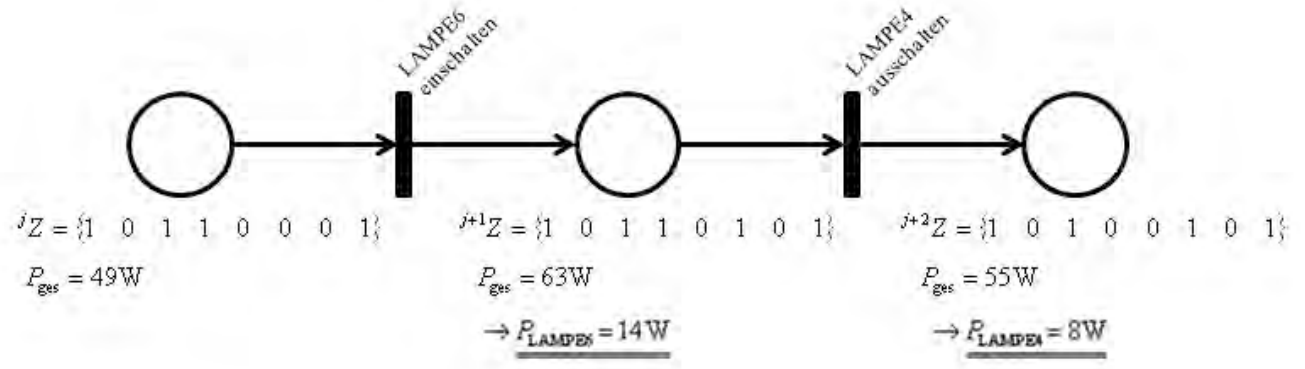

Abbildung 3: möglicher Ablauf zur Bestimmung der Leistungsaufnahmen von LAMPE6 und LAMPE4

\section{Zusammenfassung und Ausblick}

Das in diesem Beitrag vorgestellte SmallCAN-System bietet die Möglichkeit eine hohe Anzahl an Gerätekomponenten über einheitliche Buskoppler kostengünstig zu vernetzen und ist hinsichtlich der Leistungsaufnahme und der Investitionskosten erheblich günstiger ausgelegt als vergleichbare Systeme. Es eignet sich daher für eine dezentrale Energieverbrauchsmessung einzelner an das System angeschlossener Verbraucher. Der dazu notwendige Auswerte-Algorithmus sowie ein praktischer minimaler Testaufbau wurden vorgestellt. Die Ermittlung der Einzelleistungen aller im Testaufbau vorhandenen Verbraucher konnte erfolgreich durchgeführt werden.

Insbesondere bei einer erhöhten Anzahl an Verbrauchern im System ist der Einsatz eines dezentralen Systems mit kostengünstigen Hardwareeinheiten für die Zustandserfassung in Kombination mit der einmaligen Installation eines Energiezählers hinsichtlich der geringen Investitions-, Installations- und Betriebskosten sinnvoll.

Potenzial zur Weiterentwicklung der geschaffenen Grundlagen besteht vor allem in dem Bereich der Signalverarbeitung. Zum Einen können durch eine entsprechende Filterung, beispielsweise einem Kalman-Filter, die Daten zur Energieaufnahme eines Verbrauchers validiert und Angaben zur Verlässlichkeit der Messgrößen gewonnen werden. Weiterhin besteht durch eine Signalanalyse die Möglichkeit, Angaben zum Verhalten einzelner Verbraucher zu treffen, um später auch Verbraucher mit variabler Energieaufnahme (wie zum Beispiel Klimageräte) identifizieren zu können. Dies gewinnt 
insbesondere durch steigende Tendenzen im Bereich der intelligenten Energieüberwachung an Bedeutung, deren Ziel unter anderem die Identifikation und Optimierung des Verbraucherverhaltens gesamter Haushalte bzw. Stadtquartiere ist.

\section{Literaturverzeichnis}

[1] Schrom, H.: "Optimiertes Feldbussystem”, VDM Verlag, 2007.

[2] Schrom H.: "Realisierung eines optimierten Feldbussystems und Modellierung mit Petrinetzen", Docotral Dissertation, Techniche Universität Braunschweig, 2003.

[3] Diekhake, P.; Liu, J.; Schnieder, E: „Buslast- und Schaltungssimulation zur Validierung des optimierte Feldbussystems SmallCAN bei maximaler Auslastung“, KommA 2011, Magdeburg 2011

[4] Diekhake, P.; Fähndrich, E.; Schnieder, E.; Becker, U.:" SmallCAN: Integrierte Gebäudeautomatisierung durch einheitliches low-Power, low-Cost, OpenSource Feldbussystem“, Automation 2011, Baden-Baden, 2011

[5] Bergman, D.C.; Dong Jin; Juen, J.P.; Tanaka, N.; Gunter, C.A.; Wright, A.K.: „Distributed nonintrusive load monitoring“, Urbana, 2011 